\title{
Characterizing graph classes using twin vertices of regular induced subgraphs
}

\author{
Terry A. McKeE
}

\begin{abstract}
Being a weakly chordal graph is conjectured to be equivalent to twin vertices existing in every nontrivial regular induced subgraph. Being a split graph is easily characterized by every two vertices being twins in every regular induced subgraph (and this characterizes being chordal if the regular induced subgraphs are required to be connected). The new, intermediate graph class that consists of the graphs in which every vertex has a twin in every nontrivial regular induced subgraph is introduced and explored.
\end{abstract}

AMS 2000 SUBJECT CLASSIFICATIONS: 05C75.

KEYWORDS AND PHRASES: Twin vertices, induced regular subgraph, chordal graph, weakly chordal graph, split graph.

\section{Induced and regular induced subgraph characterizations}

It is very common to characterize graph classes in terms of properties of all induced subgraphs, as in Chapter 7- "Forbidden Subgraphs" - of the standard survey [1] of graph classes. The present paper considers, along with an emergent importance of twin vertices, how different things become when using only regular induced subgraphs. Focusing on regular induced subgraphs admittedly contrasts with computational considerations (see [4, $6,8]$, for instance).

We will first present motivation from the widely-studied classes of weakly chordal graphs and of split graphs toward a predictable new class in Section 2 that is potentially in between weakly chordal and split. Section 3 will consider the further limitation to connected regular induced subgraphs, including novel characterizations of chordal graphs.

Let $N(v)$ denote the open neighborhood set of all neighbors of a vertex $v$, and let $N[v]$ denote the closed neighborhood set $N(v) \cup\{v\}$. Define two vertices $v$ and $w$ to be twin vertices (or simply twins) in a graph if both $N(v) \subseteq N[w]$ and $N(w) \subseteq N[v]$. Notice that $v$ and $w$ are adjacent twins if and only if $N[v]=N[w]$ and are nonadjacent twins if and only if $N(v)=$ $N(w)$. A graph $G$ is $k$-regular if every $v \in V(G)$ has degree $\operatorname{deg}(v)=k$, and 
$G$ is regular if it is $k$-regular for some $k \geq 0$ (the edgeless graphs $\overline{K_{n}}$ are the 0-regular graphs). A graph is nontrivial if it has at least two vertices. If $S \subseteq V(G)$ and $v \in V(G)$, let $G[S]$ denote the subgraph of $G$ that is induced by $S$, and let $G-S=G[V(G)-S]$ and $G-v=G-\{v\}$. If $e \in E(G)$, let $G-e$ denote the subgraph of $G$ resulting from deleting the edge $e$. Denote the cycle and the path on $n$ vertices by, respectively, $C_{n}$ and $P_{n}$ (so $P_{n}$ is the path of length $n-1)$. Let $\bar{G}$ denote the complement of $G$.

A graph $G$ is weakly chordal (sometimes called weakly triangulated) if neither $G$ nor $\bar{G}$ contains an induced cycle $C_{k}$ with $k \geq 5$; see $[1,5,7]$ for additional characterizations. Note that the class of weakly chordal graphs is closed under taking induced subgraphs and under graph complementation.

Since $C_{n}$ and $\bar{C}_{n}$ have no twins with $n \geq 5$, if every nontrivial regular induced subgraph of a graph contains twin vertices, then the graph must be weakly chordal. The following new conjecture would assert the converse. But, in spite of its attractive simplicity, resolving (proving?) the "only if" direction seems surprisingly hard; for one thing, reasoning with regular induced subgraphs is hostile to attempts to use induction. (Conjecture 1.1 will not be used elsewhere in this paper, except as motivation in Section 2.)

Conjecture 1.1. A graph is weakly chordal if and only if, in every nontrivial regular induced subgraph, some two vertices are twins.

A graph $G$ is a split graph if $V(G)$ can be partitioned into $Q \cup I$ where $G[Q]$ is complete and $G[I]$ is edgeless. References $[1,5,7]$ contain additional characterizations of split graphs, one of which is that no induced subgraph is isomorphic to $2 K_{2}=\overline{C_{4}}, C_{4}$ or $C_{5}$; see [3]. Note that the class of split graphs is closed under taking induced subgraphs and under graph complementation.

Theorem 1.1. Each of the following is equivalent to being a split graph:

(1) Every regular induced subgraph is complete or edgeless.

(2) In every regular induced subgraph, every two vertices are twins.

(3) In every regular induced subgraph, some vertex is a twin of all the other vertices.

Proof. First suppose $H$ is a $k$-regular induced subgraph of a split graph $G$ (toward showing that $H$ is complete or edgeless). Suppose $V(H)$ is partitioned into $Q \cup I$ where $H[Q]$ is complete and $H[I]$ is edgeless. If $k=0$, then $H$ is edgeless, as in (1); hence, assume that $k>0$. If $I=\emptyset$, then $H$ is complete, as in (1); hence, assume the existence of vertex $v \in I$. Therefore, $N(v) \subseteq Q$ and $|N(v)|=k \leq|Q|$. If $w$ is any neighbor of $v$, then $w \in Q$ and (since $H$ is $k$-regular and $w$ is adjacent to every vertex in $Q$ as well as to $v$ in $I) \operatorname{deg}(w)=k \geq|Q|$. Therefore, $k=|Q|$, and so each vertex of $Q$ is 
adjacent to a unique vertex of $I$. But then $k=\operatorname{deg}(v)=|Q|$ implies that $v$ is adjacent to every vertex of $Q$, so $|I|=1$ and $H$ is complete, as in (1).

Next, observe that condition (1) implies (2), and that (2) implies (3).

Finally, suppose condition (3) holds, yet $G$ is not split (arguing by contradiction). Thus $G$ contains an induced subgraph $H$ isomorphic to $2 K_{2}$,

$C_{4}$, or $C_{5}$. But each possibility would contradict that $H$ is a regular induced subgraph with some vertex that is the twin of all the others.

\section{The new graph class}

The conjectured characterization of weakly chordal graphs in Conjecture 1.1 involves two existential quantifications (for some $v$ and for some $w \neq v$, vertices $v$ and $w$ are twins), and so could be called an $\exists \exists$ characterization. Similarly, characterization (2) of split graphs in Theorem 1.1 involves two universal quantifications (for each $v$ and for each $w \neq v$, vertices $v$ and $w$ are twins), and so could be called an $\forall \forall$ characterization, while (3) (for some $v$, for each $w \neq v$, vertices $v$ and $w$ are twins) could be called an $\exists \forall$ characterization. In contrast, the remainder of this paper will primarily study the new graph class defined by using the $\forall \exists$ condition "for each $v$, for some $w \neq v$, vertices $v$ and $w$ are twins."

For the purposes of this paper, provisionally define a graph to be regularly hereditarily $\forall \exists$-twinned — or $r h-\forall \exists$-twinned for short —if, in every nontrivial regular induced subgraph $H$, every vertex of $H$ has a twin in $H$. (A logician would pronounce $\forall \exists$ as "A E" in this context.) Note that the class of rh- $\forall \exists$ twinned graphs is closed under taking induced subgraphs and under graph complementation.

These rh- $\forall \exists$-twinned graphs are always weakly chordal by the easy direction of Conjecture 1.1, and split graphs are always rh- $\forall \exists$-twinned by Theorem 1.1(2). The 4-cycle $C_{4}$ is an example of an rh- $\forall \exists$-twinned graph that is not a split graph. Figure 1 shows an example of a weakly chordal graph that is not rh- $\forall \exists$-twinned - it is itself regular with neither of the two center ("square") vertices having a twin. This graph, along with its 5-connected complement, would need to be included in the list of forbidden induced subgraphs for the class of rh- $\forall \exists$-twinned graphs (along with infinitely many other complementary pairs, including $C_{n}$ and $\overline{C_{n}}$ when $n \geq 5$ ).

The following three lemmas concerning nontrivial regular subgraphs will build toward Theorem 2.1, which will show how the rh- $\forall \exists$-twinned graphs can be characterized with the scope of the universal quantifier greatly restricted. In any graph, let $N_{2}^{\sharp}(v)$ denote the set of vertices that are at distance exactly 2 from a vertex $v$. 


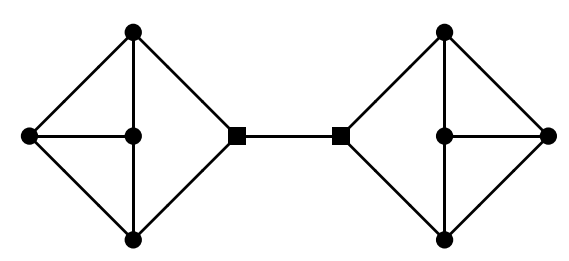

Figure 1: A weakly chordal graph that is not $\mathrm{rh}-\forall \exists$-twinned.

Lemma 2.1. Suppose $H$ is a nontrivial regular graph such that vertices in induced $P_{4}$ subgraphs of $H$ always have twins in $H$. If a vertex $v$ has no twin in $H$, then $V(H)$ is partitioned into $\{v\} \cup N(v) \cup N_{2}^{\sharp}(v)$, each vertex $x \in N(v)$ is nonadjacent with a vertex in $N(v)-\{x\}$ and has a neighbor in $N_{2}^{\sharp}(v)$, and each vertex $z \in N_{2}^{\sharp}(v)$ is nonadjacent with a vertex in $N(v)$ and has a neighbor in $N_{2}^{\sharp}(v)$.

Proof. Suppose $H$ is a nontrivial $k$-regular graph such that vertices in induced $P_{4}$ subgraphs of $H$ always have twins in $H$, and suppose $v \in V(H)$ has no twin in $H$. Thus $k \geq 2$ and $v$ is not in an induced $P_{4}$ of $H$, and so $V(H)$ is partitioned into $\{v\} \cup N(v) \cup N_{2}^{\sharp}(v)$.

Suppose $x \in N(v)$. If $N(x) \subseteq N[v]$, then $H$ being $k$-regular would imply $\operatorname{deg}(v)=\operatorname{deg}(x)$ and so $N[x]=N[v]$ (contradicting that $v$ has no twin in $H)$. Therefore, $N(x) \nsubseteq N[v]$, making $x$ adjacent to some vertex in $N_{2}^{\sharp}(v)$ and nonadjacent with some vertex in $N(v)-\{x\}$.

Now suppose $z \in N_{2}^{\sharp}(v)$. If $N(z) \subseteq N(v)$, then $H$ being $k$-regular would imply $\operatorname{deg}(v)=\operatorname{deg}(z)$ and so $N(z)=N(v)$ (contradicting that $v$ has no twin in $H)$. Therefore, $N(z) \nsubseteq N(v)$, making $z$ adjacent to some vertex in $N_{2}^{\sharp}(v)$ and nonadjacent with some vertex in $N(v)-\{x\}$.

Lemma 2.2. Suppose $H$ is a nontrivial regular graph such that vertices in induced $P_{4}$ subgraphs of $H$ always have twins in $H$. If a vertex $v$ has no twin in $H$, then the subgraph $\bar{H}[N(v)]$ of the complement of $H$ will not be connected and $H[N(v)]$ will be connected.

If $V_{1}, \ldots, V_{r}$ are subsets of $N(v)$ such that $\bar{H}\left[V_{1}\right], \ldots, \bar{H}\left[V_{r}\right]$ are the connected components of $\bar{H}[N(v)]$, then $r \geq 2$ and, for each $i$, all the vertices of $V_{i}$ will have the same neighbors in $N_{2}^{\sharp}(v)$ and the same degree-denote it by $d_{i}$-inside the subgraph $H\left[V_{i}\right]$.

Proof. Suppose $H$ is a nontrivial $k$-regular graph such that vertices in induced $P_{4}$ subgraphs of $H$ always have twins in $H$, and suppose $v \in V(H)$ has no twin in $H$. In this proof, neighbors and the $N(\cdot)$ notation will always be in terms of $H(\operatorname{not} \bar{H})$. 
If $x, y \in N(v)$ are nonadjacent and $z \in N_{2}^{\sharp}(v) \cap[N(x)-N(y)]$, then $z, x, v, y$ would be an induced $P_{4}$ containing $v$ (contradicting that $v$ has no twin in $H)$. Thus nonadjacent vertices in $N(v)$ must have exactly the same neighbors in $N_{2}^{\sharp}(v)$. Because of this, if $\bar{H}[N(v)]$ were a connected subgraph of $\bar{H}$, then all the vertices in $N(v)$ would have exactly the same neighbors in $N_{2}^{\sharp}(v)$. By the definition of $N_{2}^{\sharp}(v)$, each $z \in N_{2}^{\sharp}(v)$ has at least one neighbor in $N(v)$ and so would have $N(v)=N(z)$ (contradicting that $v$ has no twin in $H)$. Therefore, $\bar{H}[N(v)]$ is not connected, and so its complement $H[N(v)]$ must be connected.

Let $V_{1}, \ldots, V_{r}$ be the subsets of $N(v)$ such that $\bar{H}\left[V_{1}\right], \ldots, \bar{H}\left[V_{r}\right]$ are the connected components of $\bar{H}[N(v)]$, noting that $r \geq 2$ (since $\bar{H}[N(v)]$ is not connected) and each $\left|V_{i}\right| \geq 2$ (since each vertex in $N(v)$ is on an edge of $\bar{H}[N(v)]$ by Lemma 2.1). For each $i \in[1, r]$, the preceding paragraph shows that all the vertices of $V_{i}$ will have the same neighbors in $N_{2}^{\sharp}(v)$ and, since $H$ is regular, they will all have the same degree inside $H\left[V_{i}\right]$.

Lemma 2.3. Suppose $H$ is a nontrivial regular graph such that vertices in induced $P_{4}$ subgraphs of $H$ always have twins in $H$. If a vertex $v$ has no twin in $H$, then $H\left[N_{2}^{\sharp}(v)\right]$ will not be connected and the subgraph $\bar{H}\left[N_{2}^{\sharp}(v)\right]$ of the complement of $H$ will be connected.

If $V_{1}^{\prime}, \ldots, V_{r^{\prime}}^{\prime}$ are the subsets of $N_{2}^{\sharp}(v)$ such that $H\left[V_{1}^{\prime}\right], \ldots, H\left[V_{r^{\prime}}^{\prime}\right]$ are the connected components of $H\left[N_{2}^{\sharp}(v)\right]$, then $r^{\prime} \geq 2$ and, for each $i$, all the vertices of $V_{i}^{\prime}$ will have the same neighbors in $N(v)$ and the same degreedenote it by $d_{i}^{\prime}$-inside the subgraph $H\left[V_{i}^{\prime}\right]$.

Proof. Suppose $H$ is a nontrivial $k$-regular graph such that vertices in induced $P_{4}$ subgraphs of $H$ always have twins in $H$, and suppose $v \in V(H)$ has no twin in $H$. In this proof, neighbors and the $N(\cdot)$ notation will always be in terms of $H(\operatorname{not} \bar{H})$.

If $z, w \in N_{2}^{\sharp}(v)$ are adjacent and $x \in N(v) \cap[N(z)-N(w)]$, then $w, z, x, v$ be an induced $P_{4}$ containing $v$ (contradicting that $v$ has no twin in $H$ ). Thus adjacent vertices in $N_{2}^{\sharp}(v)$ must have exactly the same neighbors in $N(v)$. Because of this, if $H\left[N_{2}^{\sharp}(v)\right]$ were a connected subgraph of $H$, then all the vertices in $N_{2}^{\sharp}(v)$ would have exactly the same neighbors in $N(v)$. By Lemma 2.1, each $x \in N(v)$ has at least one neighbor $z$ in $N_{2}^{\sharp}(v)$, and so $N(v)=N(z)$ (contradicting that $z$ is not a twin of $v$ in $H$ ). Therefore, $H\left[N_{2}^{\sharp}(v)\right]$ is not connected, and so its complement $\bar{H}\left[N_{2}^{\sharp}(v)\right]$ must be connected.

Let $V_{1}^{\prime}, \ldots, V_{r^{\prime}}^{\prime}$ be the subsets of $N_{2}^{\sharp}(v)$ such that $H\left[V_{1}^{\prime}\right], \ldots, H\left[V_{r^{\prime}}^{\prime}\right]$ are the connected components of $H\left[N_{2}^{\sharp}(v)\right]$, noting that $r^{\prime} \geq 2$ (since $H\left[N_{2}^{\sharp}(v)\right]$ 
is not connected) and each $\left|V_{i}^{\prime}\right| \geq 2$ (since each vertex in $N_{2}^{\sharp}(v)$ is on an edge of $H\left[N_{2}^{\sharp}(v)\right]$ by Lemma 2.1). For each $i \in\left[1, r^{\prime}\right]$, the preceding paragraph shows that all the vertices of $V_{i}^{\prime}$ will have the same neighbors in $N(v)$ and, since $H$ is regular, they will all have the same degree inside $H\left[V_{i}^{\prime}\right]$.

Theorem 2.1. A graph is regularly hereditarily $\forall \exists$-twinned if and only if, in every nontrivial regular induced subgraph $H$, every vertex that is in an induced $P_{4}$ subgraph of $H$ has a twin in $H$.

Proof. The only-if direction follows immediately from the definition of rh$\forall \exists$-twinned graphs.

For the converse, suppose that $G$ is an arbitrary graph such that, in every nontrivial regular induced subgraph, every vertex that is in an induced $P_{4}$ subgraph has a twin. But also assume that $G$ is not rh- $\forall \exists$-twinned (arguing by contradiction). Thus $G$ must contain a particular nontrivial $k$-regular induced subgraph $H$ that contains a vertex $v$ that has no twin in $H$ (and so $v$ is not in an induced $P_{4}$ of $H$ ).

In the notation of Lemmas 2.1, 2.2, and 2.3, if $i \neq j$, then $H$ contains all $V_{i}$-to- $V_{j}$ edges and no $V_{i}^{\prime}$-to- $V_{j}^{\prime}$ edges. Also, for each $i \in[1, r]$, there is a subset $S(i) \subseteq\left[1, r^{\prime}\right]$ such that $H$ contains all $V_{i}$-to- $V_{j}^{\prime}$ edges when $j \in S(i)$ and no $V_{i}$-to- $V_{j}^{\prime}$ edges when $j \notin S(i)$; in fact, $\emptyset \neq S(i) \subset\left[1, r^{\prime}\right]$, since Lemma 2.1 shows that each vertex in $N(v)$ is adjacent to a vertex in $N_{2}^{\sharp}(v)$ and each vertex in $N_{2}^{\sharp}(v)$ is nonadjacent with a vertex in $N(v)$. The remainder of this proof will show that the existence of such $V_{1}, \ldots, V_{r}$ and $V_{1}^{\prime}, \ldots, V_{r^{\prime}}^{\prime}$-along with $d_{1}, \ldots, d_{r} ; d_{1}^{\prime}, \ldots, d_{r^{\prime}}^{\prime}$ and $S(1), \ldots, S(r)$ - is inconsistent with $H$ being $k$-regular.

For each $i$, let $n_{i}=\left|V_{i}\right|$ and $n_{i}^{\prime}=\left|V_{i}^{\prime}\right|$, noting that each $d_{i}<n_{i}$ and each $d_{i}^{\prime}<n_{i}^{\prime}$. Since $|N(v)|=k$ and $N(v)$ is partitioned into $V_{1} \cup \cdots \cup V_{r}$,

$$
k=n_{1}+\cdots+n_{r}
$$

holds. Since the open neighborhood in $H$ of each vertex in $V_{i}$ consists of the vertex $v$, the $n_{j}$ vertices in each $V_{j}$ for which $i \neq j$, the $n_{j}^{\prime}$ vertices in each $V_{j}^{\prime}$ for which $j \in S(i)$, and $d_{i}$ of the vertices in $V_{i}$ itself, $H$ being $k$-regular implies that

$$
k=1+\sum_{j \neq i} n_{j}+\sum_{j \in S(i)} n_{j}^{\prime}+d_{i}
$$

holds for each $i \in[1, r]$. Since the open neighborhood in $H$ of each vertex in $V_{j}^{\prime}$ consists of the $n_{i}$ vertices in each $V_{i}$ for which $j \in S(i)$ and $d_{j}^{\prime}$ of the 
vertices in $V_{j}^{\prime}$ itself (and rewriting $j \in S(i)$ as $i \in S^{-}(j)$ ), $H$ being $k$-regular implies that

$$
k=\sum_{i \in S^{-}(j)} n_{i}+d_{j}^{\prime}
$$

holds for each $j \in\left[1, r^{\prime}\right]$. Combining equations (1) and (2) (canceling the terms $n_{i}$ that have $j \neq i$ ) shows that $n_{i}=1+\sum_{j \in S(i)} n_{j}^{\prime}+d_{i}$ holds for each $i \in[1, r]$, and so that

$$
n_{i}=1+\sum_{h \in S(i)} n_{h}^{\prime}+d_{i}
$$

holds for each $i \in[1, r]$. Similarly combining equations (1) and (3) (canceling the terms $n_{i}$ that have $\left.i \in S^{-}(j)\right)$ shows that $\sum_{i \notin S^{-}(j)} n_{i}=d_{j}^{\prime}$ holds for each $j \in\left[1, r^{\prime}\right]$, and so (since $d_{j}^{\prime}<n_{j}^{\prime}$ ) that

$$
\sum_{i \notin S^{-}(j)} n_{i}<n_{j}^{\prime}
$$

holds for each $j \in\left[1, r^{\prime}\right]$. Then substituting the expressions for $n_{i}$ when $i \notin S^{-}(j)$ from (4) into (5) shows that

$$
\sum_{i \notin S^{-}(j)} 1+\sum_{i \notin S^{-}(j)} \sum_{h \in S(i)} n_{h}^{\prime}+\sum_{i \notin S^{-}(j)} d_{i}<n_{j}^{\prime}
$$

holds for each $j \in\left[1, r^{\prime}\right]$.

Consider the final inequality that would result from adding the inequalities in (6) over all $j \in\left[1, r^{\prime}\right]$. No such $j$ could be in every $S(i)$ (otherwise some $z \in V_{j}^{\prime}$ with $j \in S(i)$ would be a twin of $v$, contradicting that $v$ has no twin in $H$ ), while each $S(i)$ would contain some $h \in\left[1, r^{\prime}\right]$. Therefore, each $n_{j}^{\prime}$ with $j \in\left[1, r^{\prime}\right]$ would occur at least once as a term on the left side of that final inequality (contradicting that the strictly larger right side would be $n_{1}^{\prime}+\cdots+n_{r^{\prime}}^{\prime}$.

A graph is a cograph (short for complement reducible graph) if it has no induced subgraph isomorphic to $P_{4}$; see $[1,2,5,7]$ for additional characterizations. Note that the class of cographs is closed under taking induced subgraphs and under graph complementation (since $\overline{P_{4}} \cong P_{4}$ ). Corollary 2.1 is an immediate consequence of Theorem 2.1.

Corollary 2.1. Every cograph is regularly hereditarily $\forall \exists$-twinned. 
An intriguing question related to Corollary 2.1 is whether graphs are rh$\forall \exists$-twinned if and only if all their regular induced subgraphs are cographs. Theorem 2.2 shows how that possibility would conform to the fact that all rh- $\forall \exists$-twinned graphs are weakly chordal.

Theorem 2.2. A graph is weakly chordal if and only if every induced order$k$ subgraph that is 2-regular or $(k-3)$-regular is a cograph.

Proof. First suppose $G$ is weakly chordal with an order- $k$ induced subgraph $H$ that is 2-regular or $(k-3)$-regular. If $k \in\{3,4\}$, then either $H$ or $\bar{H}$ is in $\left\{K_{3}, C_{4}, K_{4}\right\}$, and so $H$ is a cograph. If $k \geq 5$ and $H$ is 2-regular, then $H \cong C_{k}$, contradicting that $G$ is weakly chordal. Thus, assume $k \geq 6$ and $H$ is $\left(k-3\right.$ )-regular (since $\overline{C_{5}} \cong C_{5}$ and $5-3=2$ ). The 2-regular graph $\bar{H}$ must be the union of two or more vertex-disjoint cycles, each of which is a triangle or a 4-cycle (since $\bar{H}$ is weakly chordal). Therefore, $\bar{H}$ is a cograph, and so $H$ is a cograph.

Conversely, suppose $G$ is not weakly chordal, and so $G$ contains either $H \cong C_{k}$ or $H \cong \overline{C_{k}}$ with $k \geq 5$ as a nontrivial regular induced subgraph. If $H \cong C_{k}$, then $k \geq 5$ implies that $H$ is 2-regular but not a cograph. If $H \cong \overline{C_{k}}$, then $k \geq 5$ implies that $\bar{H} \cong C_{k}$ is not a cograph, so $H$ is $(k-3)$-regular but not a cograph.

\section{Connected regular induced subgraphs}

Strengthening the statements of Theorems 1.1 and 2.1 to involve all connected regular induced subgraphs leads to several related results. First notice that Conjecture 1.1 is equivalent to a graph being weakly chordal if and only if, in every nontrivial connected regular induced subgraph, some two vertices are twins. In contrast to that $\exists \exists$ formulation, Theorem 3.1 will show that the corresponding $\forall \forall$ and $\exists \forall$ results now characterize the class of chordal graphs (instead of the class of split graphs).

A graph $G$ is chordal if $G$ contains no induced cycle $C_{k}$ with $k \geq 4$. References $[1,5,7]$ contain additional characterizations of chordal graphs, one of which is that every induced subgraph contains a simplicial vertex (meaning a vertex $v$ such that $G[N[v]]$ is complete). Note that the class of chordal graphs is closed under taking induced subgraphs (but not under graph complementation, since $2 K_{2} \cong \overline{C_{4}}$ is chordal). The graph $2 K_{2}$ and the connected graph that consists of two triangles with one shared vertex are chordal graphs that are not split graphs and that do not satisfy any of the three conditions in Theorem 3.1.

Theorem 3.1. Each of the following is equivalent to a graph being chordal: 
(1) Every connected regular induced subgraph is complete.

(2) In every connected regular induced subgraph, every two vertices are twins.

(3) In every connected regular induced subgraph, some vertex is a twin of all the other vertices.

Proof. First suppose $H$ is a connected $k$-regular induced subgraph of a chordal graph $G$ (toward showing that $H$ is complete). Since $H$ is also chordal, $H$ has a simplicial vertex $v$ with degree $k$ and $H[N[v]]$ complete. Each neighbor of $v$ also having degree $k$ implies $H=H[N[v]]$ is complete, as in (1).

Next, observe that condition (1) implies (2), and that (2) implies (3).

Finally, suppose condition (3) holds, yet $G$ is not chordal (arguing by contradiction). But then $G$ would contain an induced cycle $C_{k}$ with $k \geq 4$, contradicting that $C_{k}$ is a connected regular induced subgraph with no vertex that is the twin of all the other vertices.

Theorem 3.2 is an additional characterization of rh- $\forall \exists$-twinned graphs.

Theorem 3.2. A graph is regularly hereditarily $\forall \exists$-twinned if and only if, in every nontrivial connected regular induced subgraph, every vertex has a twin.

Proof. The only-if direction follows immediately from the definition of rh$\forall \exists$-twinned graphs.

For the converse, suppose $G$ is an arbitrary graph such that, in every nontrivial connected regular induced subgraph, every vertex has a twin. But also assume that $G$ is not $\forall \exists$-twinned (arguing by contradiction). Thus $G$ must contain a particular nontrivial regular induced subgraph $H$ that is not connected and contains a vertex $v$ that has no twin in $H$. Let $H_{v}$ be the connected component of $H$ that contains $v$ and let $H_{w}$ be a different connected component containing a vertex $w$. If $\left|V\left(H_{v}\right)\right|=1$, then $H$ being regular would imply that $v$ and $w$ would be isolated vertices of $H$ (contradicting that $w$ is not a twin of $v$ in $H$ ). If instead $\left|V\left(H_{v}\right)\right| \geq 2$, then $H_{v}$ would be a nontrivial connected regular induced subgraph of $H$ and so, by the assumed nature of $G$, the vertex $v$ would have a twin in $H_{v}$ (contradicting that $v$ has no twin in $H$ ).

The known direction of Conjecture 1.1 combines with Theorems 3.1 and 3.2 to show that the class of rh- $\forall \exists$-twinned graphs is sandwiched between the classes of weakly chordal graphs and chordal graphs. Theorems 3.1 and 3.2 also have the following consequence.

Corollary 3.1. Every chordal graph is regularly hereditarily $\forall \exists$-twinned. 
In closing, note that the characterizations of rh- $\forall \exists$-twinned graphs in Theorems 2.1 and 3.2 are unsatisfactory in that they involve quantification over all regular induced subgraphs. It remains highly desirable to find a characterization within the standard graph-theoretic vernacular - something more like the traditional characterizations of weakly chordal, split, and chordal graphs. Such a characterization can also be hoped to suggest a better name for the graph class than the unwieldy "regularly hereditarily $\forall \exists$-twinned" terminology.

\section{References}

[1] A. Brandstädt, V.-B. Le, and J. P. Spinrad (1999). Graph Classes: A Survey. Society for Industrial and Applied Mathematics, Philadelphia. MR1686154

[2] D. G. Corneil, H. Lerchs, and L. Stewart Burlingham (1981). Complement reducible graphs. Discrete Appl. Math. 3 163-174. MR0619603

[3] S. Földes and P. L. Hammer (1977). Split graphs having Dilworth number two. Canad. J. Math. 29 666-672. MR0463041

[4] S. Gupta, V. Raman, and S. Saurabh (2012). Maximum r-regular induced subgraph problem: Fast exponential algorithms and combinatorial bounds. SIAM J. Discrete Math. 26 1758-1780. MR3024146

[5] T. A. McKee and F. R. McMorris (1999). Topics in Intersection Graph Theory. Society for Industrial and Applied Mathematics, Philadelphia. MR1672910

[6] H. Moser and D. M. Thilikos (2009). Parameterized complexity of finding regular induced subgraphs. J. Discrete Algorithms 7 181-190. MR2526838

[7] J. P. Spinrad (2003). Efficient Graph Representations. American Mathematical Society, Providence. MR1971502

[8] S. Sridharan (2008). Polynomial time algorithms for two classes of subgraph problem. RAIRO - Operations Research 42 291-298. MR2444488

TERry A. MCKeE

Wright State University

DAYTON, OHIO 45435

USA

E-mail address: terry.mckee@wright.edu

ReCEIVED 12 MAY 2014 